\title{
Driving cycle tracking device big data storing and management
}

\author{
Arunkumar Subramaniam ${ }^{1}$, Nurru Anida Ibrahim ${ }^{1}$, Siti Norbakyah Jabar ${ }^{1,2,3}$, \\ Salisa Abdul Rahman 1,2,3 \\ ${ }^{1}$ Faculty of Ocean Engineering Technology and Informatics, Universiti Malaysia Terengganu, Terengganu, Malaysia \\ ${ }^{2}$ Renewable Energy and Power Research Interest Group (REPRIG), Universiti Malaysia Terengganu, Terengganu, Malaysia \\ ${ }^{3}$ Energy Storage Research Group (ESRG), Universiti Malaysia Terengganu, Terengganu, Malaysia
}

\begin{abstract}
Article Info
Article history:

Received Apr 16, 2021

Revised Jun 29, 2021

Accepted Jul 27, 2021

\section{Keywords:}

Cloud storage

Driving cycle

Hybrid electric vehicles

Internet of things

IR4.0

Speed-time profile

ABSTRACT

Driving cycle is commonly known as a series of speed-time profile. Research on this discipline aids vehicle manufacturing industries in vehicle manufacturing, environmentalists to study on environment quality and profile in accordance to vehicle emissions besides traffic engineers to further investigate the behavior of drivers and the conditions of roads in a certain area or cluster. This also assists automotive industries to innovate energy efficient vehicles which reduce vehicle emissions and energy wastages which lead to air pollution in which a major threat for human health according to Goal 3 of united nations (UN) sustainable development goals (SDG). To construct an accurate driving cycle, data based on real-world driving behavior is crucial and as the world is advancing in technology, the usage of internet of things (IoT) plays an important role in innovatietcons. IoT is an idea of computing every day physical object and information into computers, devices and software. These devices work by using sensors that transmit data to a computer or software allowing them to perform important tasks as needed. In this research, an idea of data collecting device, driving cycle tracking device (DC-TRAD) is constructed with implementation of IoT in which the collected data will be saved into my structured query language (MySQL) database instantly for data storing.
\end{abstract}

This is an open access article under the CC BY-SA license.

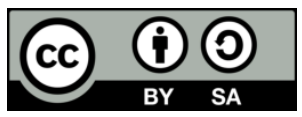

\section{Corresponding Author:}

Salisa Abdul Rahman

Faculty of Ocean Engineering Technology and Informatics, Universiti Malaysia Terengganu

21030 Kuala Nerus, Terengganu, Malaysia

Email: salisa@umt.edu.my

\section{INTRODUCTION}

In the paper, Achour et al. [1] one of the most significant issues which have been under discussion by various councils and its effect on human health is the issue of carbon emission. The paper also explained recent developments in this discipline have heightened the need for driving cycle establishment in order to redude the count of emissions emitted by vehicles into the environment. Several problems have been encountered lately which leads to the idea of driving cycle tracking device (DC-TRAD) construction. First and foremost, in order to come up with a proper and accurate driving cycle, it consumes a lot more time and man-power in order to collect required information and adequate data [2]. Driving cycle plays an important role in automotive sector where engineers requires driving cycle to analyze road condition, road profile, driving behavior, fuel consumptions, fuel emissions and road conditions and latter aids vehicle manufacturing sectors in more efficient vehicle productions [3]-[6]. Driving cycle is a profile which represents a speed-time relationship in which can be used to draw out fuel economy of a vehicle [7]-[11]. In the paper, Pelkmans et al. [12] driving cycles which is also known as test cycles can be categorized into two major categories which is time-based and distance-based. The time-based cycles can be implemented easily in which it is suitable for only a certain class 
of vehicles whereas distance-based cyces are more difficult to be implemented but suits better to reality compared to time-based cycles. To construct an accurate driving cycle, it is very time consuming to record and collect the information required which is the speed of the vehicle in line with time. Other than that, it is also important to have a stable platform to store the collected big data which is speed to be analyzed in future. Big data analytics is a topic fraught with both positive and negative potential and it is not defined just by the amount of information involved but also its variety and complexity [13]. Big data is a term used for a set of data which is large and complex in which humans have difficulties to manage and process on-hand [14], [15]. Thus, phpMyAdmin is chosen to store the collected big amount of data. phpMyAdmin is an open source tool for my structured query language (MySQL) in which it is written in hypertext prepocessor (PHP) and been one of the most popular administration tool for web hosting services.

Three different platforms are used in this research, which is espressif systems (ESP32) microcontroller, Neo-7 global positioning system (GPS) module and phpMyAdmin web server host as internet of things (IoT) platform to store data. ESP32 is used because ESP32 has two cores, one core to run wifi functions and one core to execute uploaded programs. ESP32 also has a wifi and bluetooth module, and 36 general purpose input/output (GPIO) [16]. ESP32 microcontroller is an easy-to-go microcontroller in which it can be interfaced with other components easily. ESP32 is a dual-core system which has two cores of central processing unit (CPU) which is PRO-CPU for protocol and APP-CPU for application. To add to that, this embedded system has address space of $4 \mathrm{~GB}$ and peripheral address space of $512 \mathrm{~KB}$. There are external memories in this embedded system too which are $448 \mathrm{~KB}$ read only memory (ROM), $520 \mathrm{~KB}$ static random access memory (SRAM) and two $8 \mathrm{~KB}$ real-time clock (RTC) memory and these memories support up to four times $16 \mathrm{MB}$ flash [17]. Figure 1 shows the function block diagram of ESP32 and Figure 2 shows ESP32 pinout diagram.

ESP32 Function Block Diagram

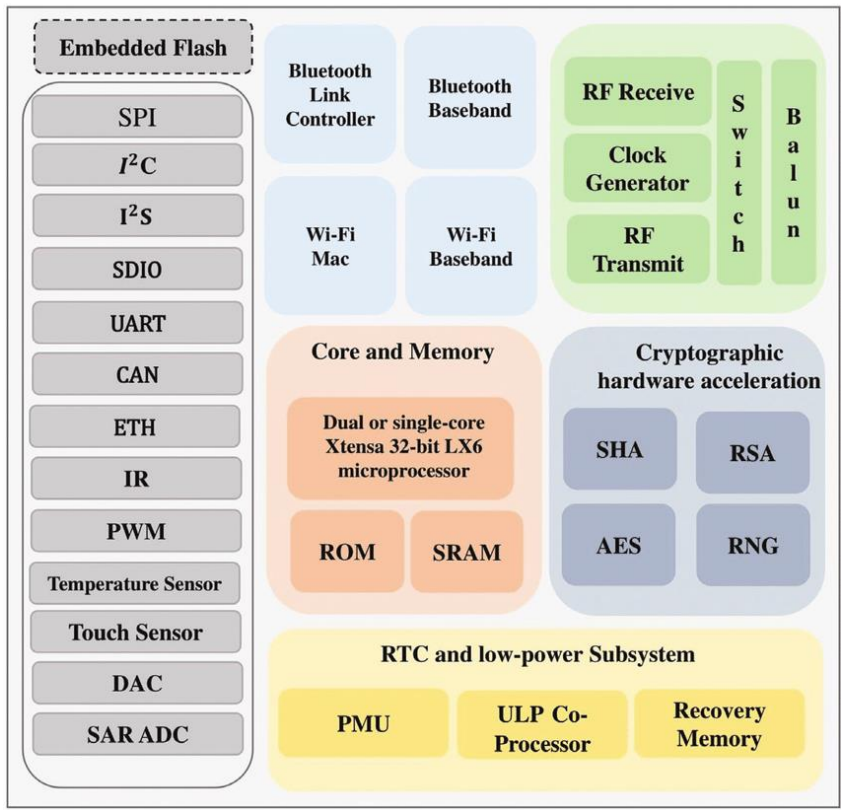

Figure 1. ESP32 function block

ESP32 is well-known for its flexibility and wide programming options it has. The common language for programming ESP32 is $\mathrm{C}$ and therefore, most application programming interface (API) libraries are provided in C. more conveniently, ESP32 can also be programmed with $\mathrm{C}++$ programming. ESP32 can also be programmed using Arduino integrated development environment (IDE) which is a great platform for students and researchers to come up with prototypes of their innovations. To aid this, an engineer from Texas, Neil Koblan, provides most of the required libraries for ESP32 APIs in his GitHub repository. Last but not least, since this microcontroller is an open source, it is very handy to develop an operating system for ESP32 and IoT platform integration [18]. According to Santos, ESP32 chip comes with 48 pins with multiple functions. She had also discussed the uses and assignments of pins of the chip in her digital publication in which analog to digital converter (ADC) and digital to analog converter (DAC) are only assigned to static pins 
but due to ESP32's multiplexing feature, any pins can be assigned in the code to be used as universal asynchronous reception and transmission (UART), inter-integrated-circuit (I2C), serial peripheral interface (SPI) and pulse width modulation (PWM). Figure 2 shoes the pinout diagram of ESP32 microcontroller [19]. Before proceeding to use ESP32 microcontroller with Arduino IDE, a few configurations have to be made. According to John Teel, the founder of predictable designs, Arduino IDE can be accommodated with many different processor modules in addition to actual Arduino boards [20]. In accordance, ESP32 board should be added into Arduino IDE preferences tab as per steps: i) in the Arduino IDE, go to File->Preferences, and in the additional boards manager uniform resource locator (URLs) $\rightarrow$ input field, type: https://dl.espressif.com/dl/package_esp32_index.json; ii) go to Tools->Board "current board selection" -> board manager. In the pop-up window, scroll down to the bottom, and install the ESP32 by espressif systems boards.

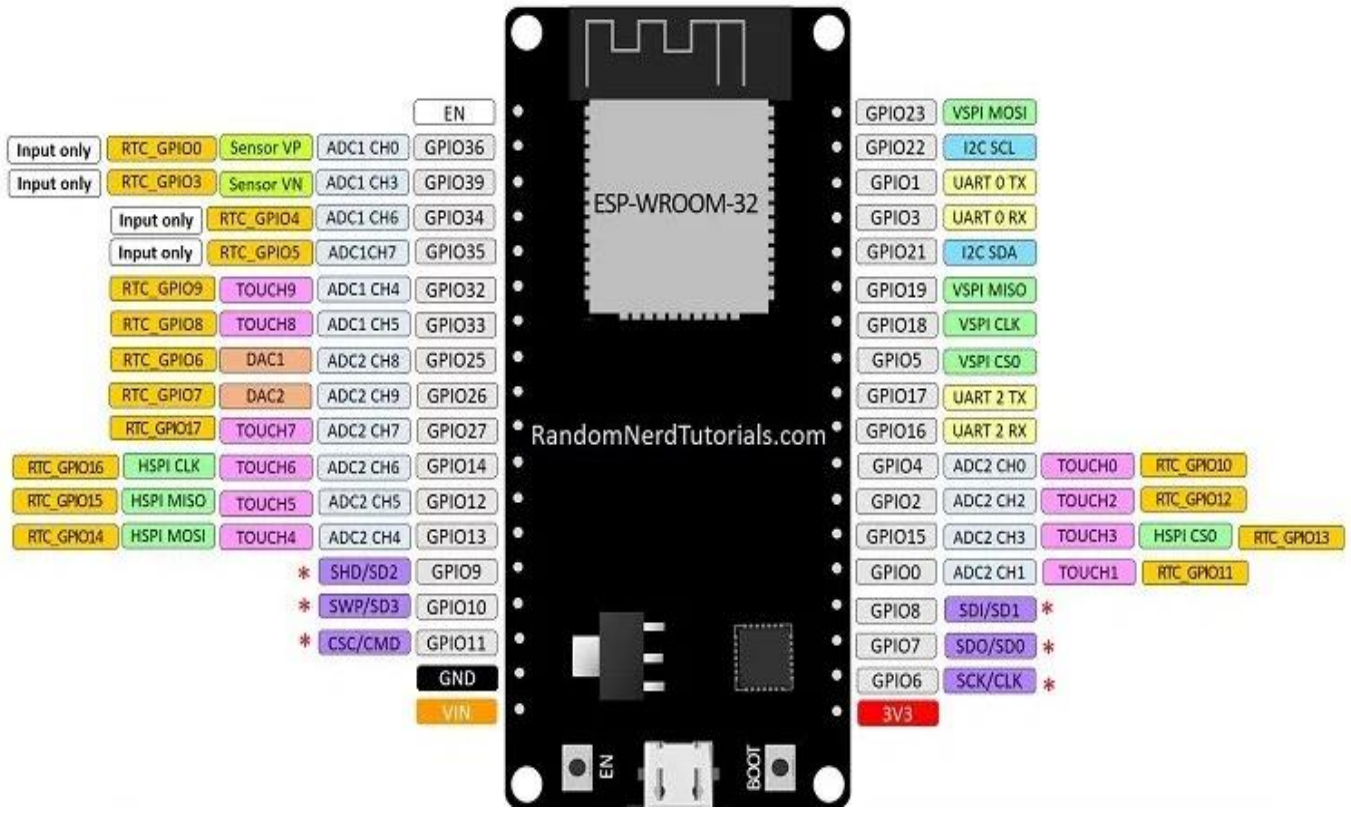

Figure 2. ESP32 pinout diagram

Second platform which is in use is NEO-7 GPS module. The GPS is a satellite-based navigation system that was developed as a military application in the $1970 \mathrm{~s}$. GPS is a one-way-ranging system; i. e. users can only receive the signals [21]. NEO-7 GPS module is a standalone global navigation satellite system (GNSS) modules which benefits from the exceptional performance of the U-BLOX 7 GNSS, GPS, navigation satellitite system (GLONASS), quasi-zenith satellite system (QZSS) and satellite-based augmentation systems (SBAS) engine. Besides, NEO-7 series delivers high sensitivity and minimal acquisition times in the industry-proven NEO form factor [22]. There are two types of NEO-7 modules which is NEO-7M and NEO-7N. NEO-7M is used for cost sensitive applications whereas NEO-7N is used widely in RF integration applications. Both NEO7 series has the ability for application of high level integration with flexible connection options in which it can be interfaced with Arduino compatible components easily. Figure 3 shows the function block diagram of NEO7 GPS series.

Lastly, phpMyAdmin plays the same important role in this research. phpMyAdmin is an administration tool which is written base don PHP and intended t handle administration and documentation of MySQL over the web server host. phpMyAdmin has vast range of supports and operations on MySQL and MariaDB. This tool is widely used by administrators to manage databases, tables, columns, indexes [23]. To increase the reliability of this web server host, phpMyAdmin has a wide range of language masters in which it is translated into 72 languages and supports both left-to-right (LTR) and right-to-left (RTL) languages. phpMyAdmin has fascinating features where it has an intuitive web interface and supports most MySQL features; browse databases, tables, fields and indexes, create and alter databases, manage structured query language (SQL) accounts, manage stored triggers and procedures, import data from comma separated values (CSV) and SQL, export data to CSV, SQL, extensible markup language (XML), portable document format (PDF), JavaScript object (JSO), international electrotechnical commission-IEC 26300, word, latex and 
administering multiple servers, graphic creations and many more [24]. Figure 4 shows the phpMyAdmin database start-up interface.

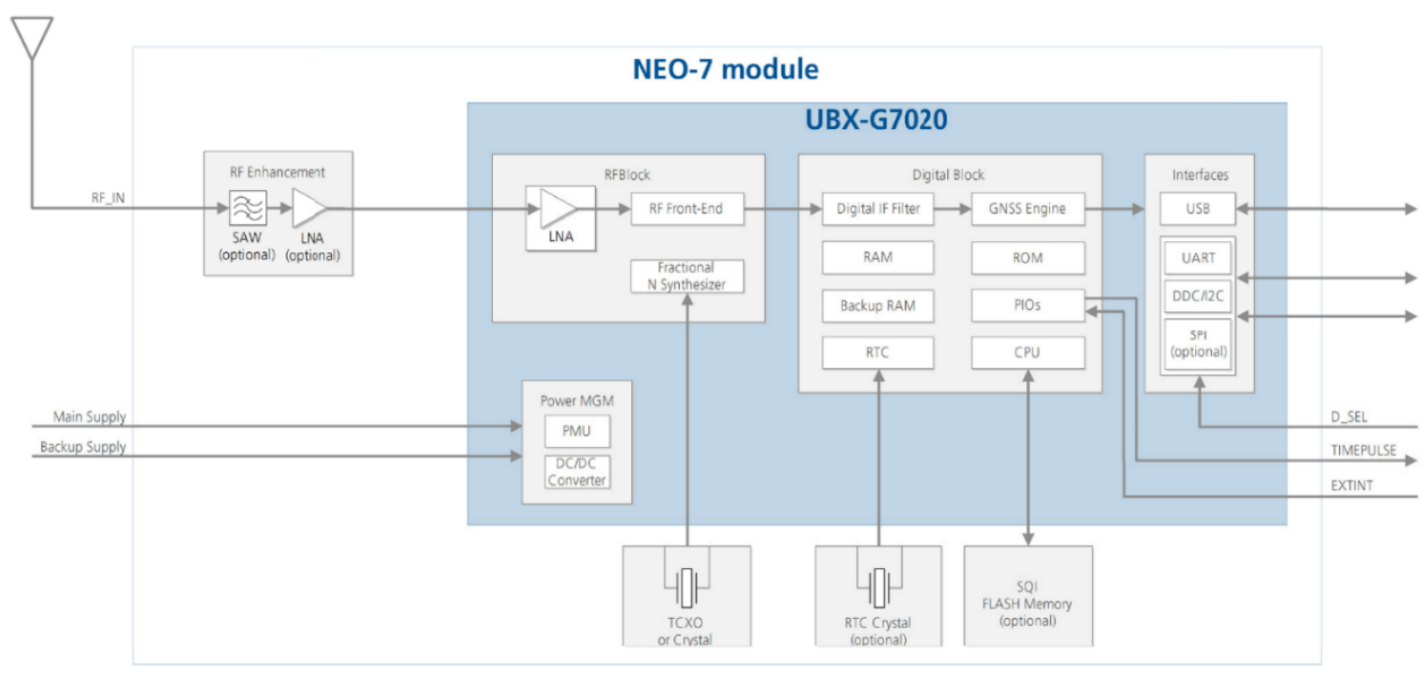

Figure 3. NEO-7 series function block

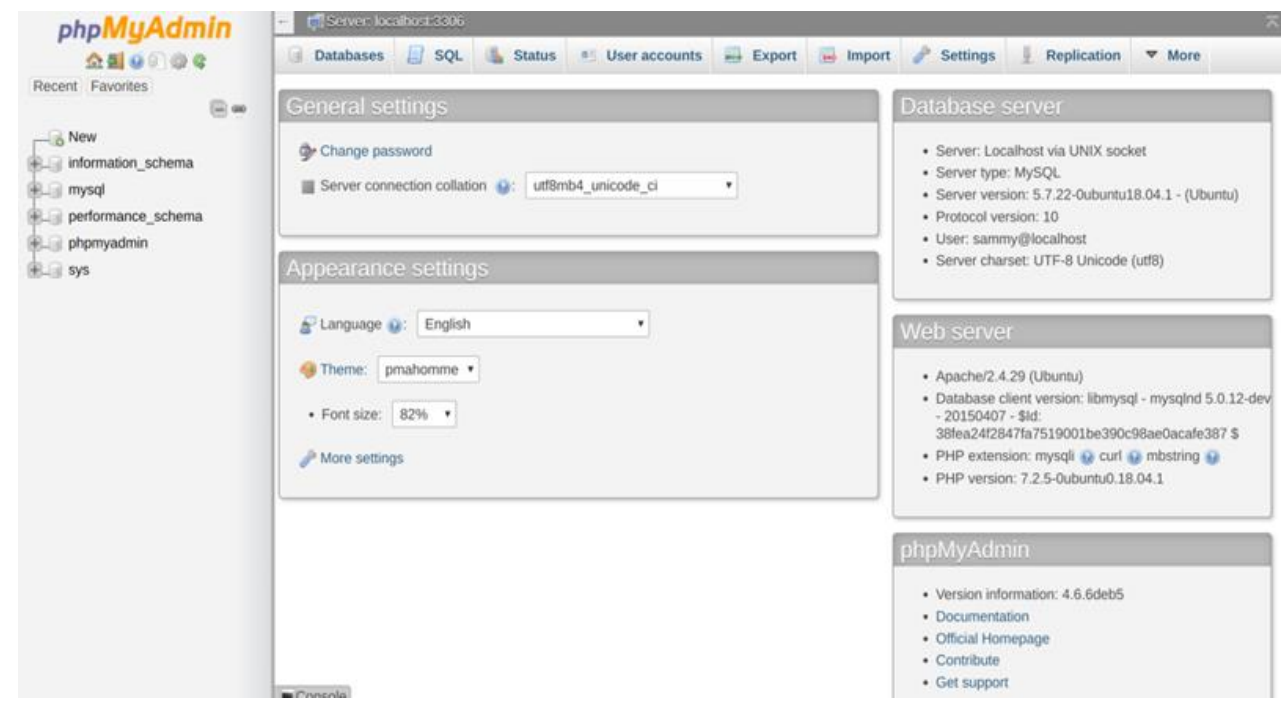

Figure 4. The phpMyAdmin database start-up interface

\section{RESEARCH METHOD}

\subsection{Component selection}

Each component used in this research were studied in detail to ensure the reliability and operability of the units. This is also to ensure proper working condition of the selected components in which short circuit can be avoided to enhance the performance of the device. Table 1 shows the list of equipment and components required.

Table 1. Lists of equipment and components

\begin{tabular}{ccc}
\hline Equipment/Components & Quantity & Remarks \\
\hline ESP32S Microcontroller & 1 & Main control unit \\
Jumper Cables & As needed & Component connections \\
TFT Screen & 1 & To display the output \\
Programming Cable & 1 & To communicate with IDE \\
GPS Shield Module & 1 & To collect parameters \\
Casing & 1 & For enclosure \\
\hline
\end{tabular}




\subsection{Prototype construction}

NEO-7M GPS module is connected to ESP32 microcontroller where the relative pins are connected as stated in Table 2. Figure 5 shows the connection diagram of NEO-7M GPS module and ESP32 microcontroller.

Table 2. DC-TRAD pin configuration

\begin{tabular}{cc}
\hline NEO-7M GPS & ESP32 \\
\hline RX & TX \\
TX & RX \\
VIN & VIN 5V \\
GND & GND \\
\hline
\end{tabular}

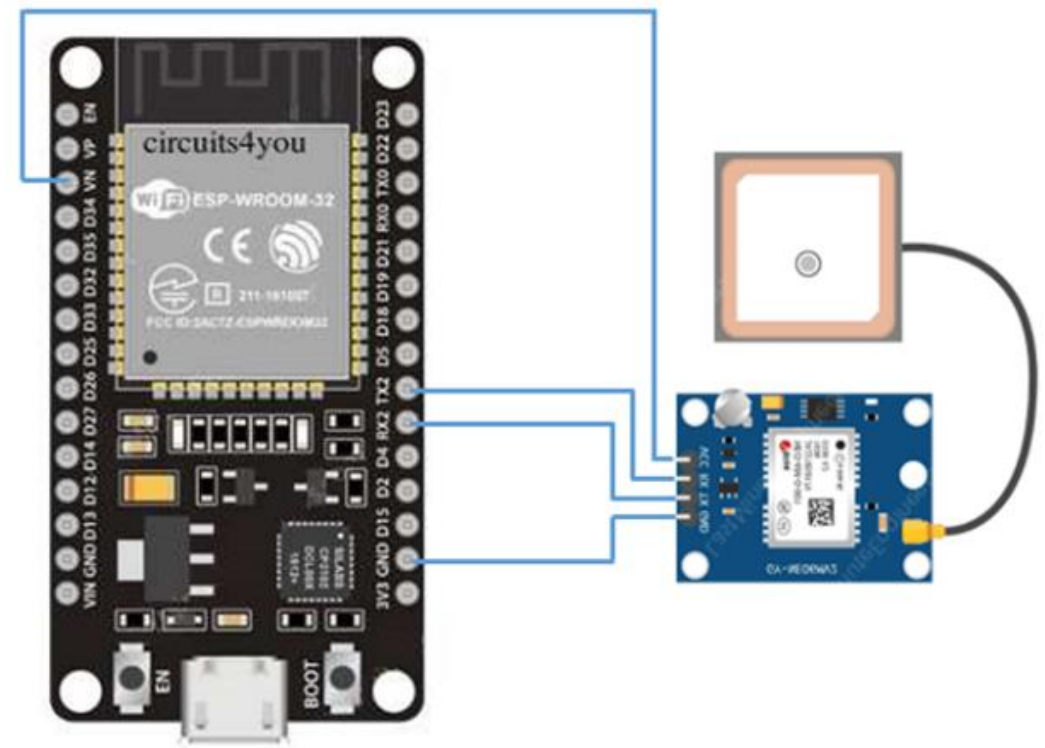

Figure 5. DC-TRAD connection diagram

\subsection{Big data storing and management}

Apart of this, big data management plays an important role in daily life in which proper management could save cost, reduce process time, gives a more precise outlook on current market conditions, maintain online reputations and standards, ability to boost-up customer monopoly, offer marketing strategies and insights and also drives new innovations. To maintain operational efficiency, big data management is also implemented in which phpMyAdmin is used with aid of XAMPP as the cross-platform to upload collected and measured data points by GPS sensor instantly into online database platform with the aid of wireless fidelity (Wi-Fi) facility provided in ESP32 microcontroller.

\section{RESULTS AND DISCUSSION}

The required components are connected as per Table 2. Figure 5 shows the connection diagram of DC-TRAD. There are a few options for power supply for DC-TRAD such as battery packs and power banks. phpMyAdmin database platform have to be created to insert information and collected data from DC-TRAD. First and foremost, a cross-platform web server is needed in order to allow WordPress offline site building on a local web server. In this research, XAMPP cross-platform is used. XAMPP is an abbreviation for crossplatform, Apache, MySQL, PHP and Perl which can be used and interfaced on Windows, Linux and also Mac easily. XAMPP is an open-source platform which can be downloaded with no cost. Figure 5 shows the interface of XAMPP. "START" option has to be chosen for Apache and MySQL to initiate the configuration. Next, choose "ADMIN" of MySQL to start up phpMyAdmin administration interface as per Figure 6 and administration platform will appear as per Figure 7.

A new database can be created according to needs from the "NEW" tab in phpMyAdmin Administration Platform. In this research, database named "dctrad" is created and a table named "gps" is 
created for data including. Upon database creation, the values in table are defined as float with a maximum of 10 digit input into database. Database and created website log can be accessed by using "localhost" internet protokol (IP) address. For device testing, DC-TRAD was allowed to run through for a time to ensure the response and update rate of the GPS sensor which is 500ms. The testing was done at Kampar, Perak with GPS coordinate of 4.2999988, 101.1499994 on 23rd February 2021 at 4.00pm allowing an error of 5\%. Figure 8 shows the website log created for DC-TRAD.

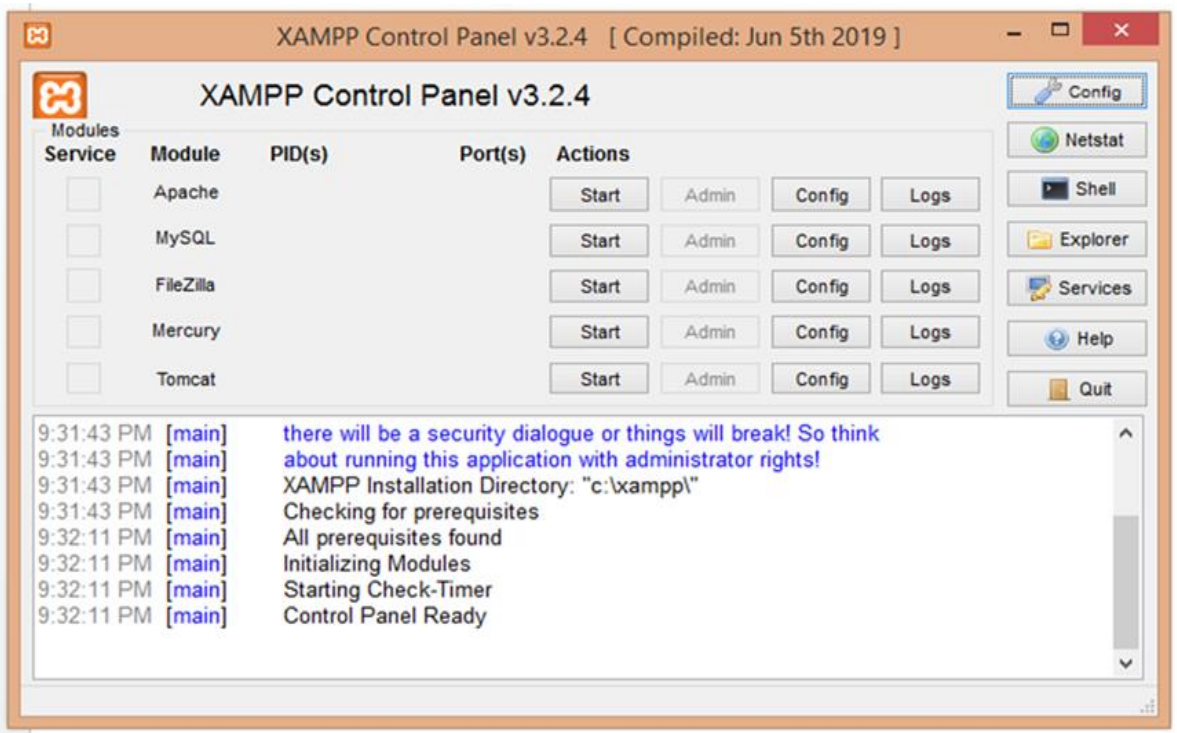

Figure 6. XAMPP open-source platform

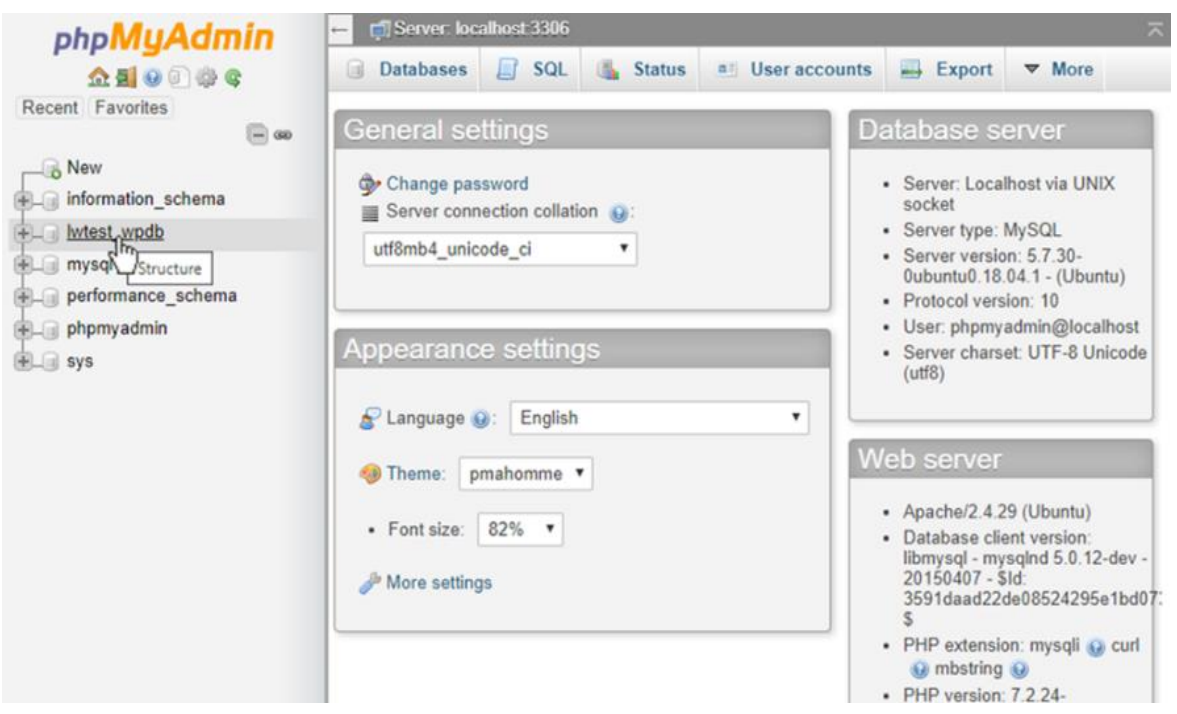

Figure 7. phpMyAdmin administration platform

Six columns are created in website log as shown in Figure 7 in which all required information will be uploaded according to the title of the column. First column named "No" is known as the data number which has AUTO_INCREMENT function. Second and third column gives latitude and longitude of the current location, and fourth column shows the travelling speed. Since the variables are declared as float function, speed data will be shown in six figures and in static position, the sensor reads some values detecting the movement of the speed of air across the GPS sensing unit. The final two columns show the current date and time which is taken according to UTC+8 Kuala Lumpur date/time. DC-TRAD is convenient to be used to collect information required to construct a detailed driving cycle as the device contains high end data management 
system in which the collected big data is uploaded into MySQL database and can be viewed from any device by using the access address of the website log anywhere and anytime. Data viewing is made easy with the aid of IoT in which collected data can be stored in Cloud platforms to be viewed any time in hand and plays an important role in technology advancement [25]. Please take note that each machine has their own unique IP address and had to be kept confidential in any circumstances. According to Shapiro, IP stands for Internet Protocol and it is also known as a unique identifier given to every machine in a network [26].

Driving Cycle Tracking Device (DC-TRAD)

\begin{tabular}{|c|c|c|c|c|c|}
\hline No & Latitude & Longitude & Speed & Date & Time \\
\hline 288 & 4.31243 & 101.154 & 1.2038 & $2021-02-23$ & $80: 84: 01$ \\
\hline 289 & 4.31236 & 101.154 & 1.62976 & $2021 \cdot 02-23$ & $68: 64: 64$ \\
\hline 290 & 4.31234 & 101.154 & 1.98164 & $2021-02-23$ & $\theta 0: 04: 06$ \\
\hline 291 & 4.31234 & 101.154 & 1.98164 & $2021=02-23$ & $60: 04: 08$ \\
\hline 292 & 4.3123 & 101.154 & 1.1112 & $2021-02-23$ & $\theta 0: 84: 11$ \\
\hline 293 & 4.3123 & 101.154 & 1.1112 & $2021-02-23$ & $60: 04: 13$ \\
\hline 294 & 4.3123 & 101.154 & 0.87044 & $2 \theta 21-\theta 2-23$ & $e \theta: e 4: 15$ \\
\hline 295 & 4.3123 & 101.154 & 0.79636 & $2 \theta 21-\theta 2-23$ & $\theta \theta: \theta 4: 18$ \\
\hline 296 & 4.31232 & 101.154 & 1.7594 & $2021-02-23$ & $\theta \theta: \theta 4: 2 \theta$ \\
\hline 297 & 4.31231 & 101.154 & 1.18528 & $2021-02-23$ & $00: 04: 22$ \\
\hline 298 & 4.3123 & 101.154 & 0.01852 & $2021-82-23$ & $68: 04: 24$ \\
\hline 299 & 4,31229 & 101.154 & 0.5556 & $2021-02-23$ & $60: 04: 27$ \\
\hline 300 & 4.31229 & 101.154 & 0.5556 & $2021-62-23$ & $68: 64: 29$ \\
\hline 301 & 4.31229 & 101.154 & 1.35196 & $2021-02-23$ & $60: 64: 32$ \\
\hline 302 & 4.31229 & 101.154 & 1.35196 & $2021-02-23$ & $60: 64: 34$ \\
\hline 303 & 4.31232 & 101.154 & 2.33352 & $2021-\theta 2-23$ & $\theta 0: 04: 36$ \\
\hline 304 & 4.31234 & 101.154 & 2.66688 & $2021-02-23$ & $\theta 0: 04: 39$ \\
\hline 305 & 4.31236 & 101.154 & 5.0004 & $2021-02-23$ & $\theta 0: 04: 41$ \\
\hline 306 & 4.31236 & 101.154 & 4.05588 & $2021-02-23$ & $\theta 0: 04: 44$ \\
\hline 307 & 4,31236 & 101.154 & 4.05588 & $2021-02-23$ & Active $\theta 0: 84: 46$ : \\
\hline 308 & 4.31236 & 101.154 & 3.9818 & $2021-02-23$ & Go $108: 84: 48$ d wase Wadom. \\
\hline 309 & 4.31237 & 101.154 & 3,4262 & $2021-02-23$ & $60: 04: 51$ \\
\hline
\end{tabular}

Figure 8. DC-TRAD website logging

\section{CONCLUSION}

To conclude, the ability of DC-TRAD as a data collector to collect required information to construct an accurate driving cycle is said to aid engineers in environment and traffic studies in which researchers are able to collect and also store big data into database with easy steps wirelessly and instantly. The flexibility of DC-TRAD is vital as the information to be collected and uploaded into database can always be managed and changed according to needs of research. As stated previously, driving cycle plays an important role among vehicle manufacturers mainly as this this device may give a hand in the early stage of driving cycle construction to collect speed and time data and can be managed systematically in MySQL database.

\section{ACKNOWLEDGEMENTS}

The authors would like to be obliged to Ministry of Education Malaysia for providing financial assistance under FRGS 2020 (59623) grant and Faculty of Ocean Engineering Technology and Informatics, UMT for all their technical and research support for this work to be successfully completed.

\section{REFERENCES}

[1] H. Achour and A. G. Olabi, "Driving cycle developments and their impacts on energy consumption of transportation," Journal of Cleaner Production, vol. 112, pp. 1778-1788, Jan. 2016, doi: 10.1016/j.jclepro.2015.08.007.

[2] P. Nyberg, E. Frisk, and L. Nielsen, "Driving Cycle Equivalence and Transformation," IEEE Transactions on Vehicular Technology, vol. 66, no. 3, pp. 1963-1974, Mar. 2017, doi: 10.1109/TVT.2016.2582079.

[3] Q. Shi, Y. B. Zheng, R. S. Wang, and Y. W. Li, “The study of a new method of driving cycles construction," Procedia Engineering, vol. 16, pp. 79-87, Jan. 2011, doi: 10.1016/J.PROENG.2011.08.1055.

[4] V. Schwarzer and R. Ghorbani, "Drive cycle generation for design optimization of electric vehicles," IEEE Transactions on Vehicular Technology, vol. 62, no. 1, pp. 89-97, Jan. 2013, doi: 10.1109/TVT.2012.2219889.

[5] N. H. Arun, S. Mahesh, G. Ramadurai, and S. M. Shiva Nagendra, "Development of driving cycles for passenger cars and motorcycles in Chennai, India," Sustainable Cities and Society, vol. 32, pp. 508-512, Jul. 2017, doi: 10.1016/j.scs.2017.05.001.

[6] S. H. Ho, Y. D. Wong, and V. W. C. Chang, "Developing Singapore Driving Cycle for passenger cars to estimate fuel consumption and vehicular emissions," Atmospheric Environment, vol. 97, pp. 353-362, Nov. 2014, doi: 10.1016/j.atmosenv.2014.08.042.

[7] C. Pan, X. Gu, L. Chen, L. Chen, and F. Yi, "Driving cycle construction and combined driving cycle prediction for fuzzy energy management of electric vehicles," International Journal of Energy Research, vol. 45, no. 12, pp. 17094-17108, Mar. 2021, doi: 10.1002/er.5320. 
[8] G. Amirjamshidi and M. J. Roorda, "Development of simulated driving cycles for light, medium, and heavy duty trucks: Case of the Toronto Waterfront Area," Transportation Research Part D: Transport and Environment, vol. 34, pp. 255-266, Jan. 2015, doi: 10.1016/j.trd.2014.11.010.

[9] I. N. Anida, I. S. Ismail, J. S. Norbakyah, W. H. Atiq, and A. R. Salisa, "Characterisation and development of driving cycle for work route in Kuala Terengganu," International Journal of Automotive and Mechanical Engineering, vol. 14, no. 3, pp. 45084517, Sep. 2017, doi: 10.15282/ijame.14.3.2017.9.0356.

[10] I. Anida and S. Abdul Rahman, "Driving cycle development for Kuala Terengganu city using k-means method," International Journal of Electrical and Computer Engineering (IJECE), vol. 9, p. 1780, 2019, doi: 10.11591/ijece.v9i3.pp1780-1787.

[11] X. Zhao, X. Zhao, Q. Yu, Y. Ye, and M. Yu, "Development of a representative urban driving cycle construction methodology for electric vehicles: A case study in Xi'an,” Transportation Research Part D: Transport and Environment, vol. 81, p. 102279, Apr. 2020, doi: 10.1016/j.trd.2020.102279.

[12] L. Pelkmans, D. De Keukeleere, H. Bruneel, and G. Lenaers, "Influence of vehicle test cycle characteristics on fuel consumption and emissions of city buses," May 2001, doi: 10.4271/2001-01-2002.

[13] K. Adam, M. Adam, I. Fakharaldien, J. Mohamad Zain, and M. Majid, "Big Data Management and Analysis,” 2014.

[14] O. Abiodun and E. O. Ademola, "A review of big data management, benefits and challenges," Journal of Emerging Trends in Computing and Information Sciences, vol. 5, no. 6, pp. 433-438, 2014.

[15] H. E. Pence, "What is Big Data and Why is it Important?," Journal of Educational Technology Systems, vol. 43, no. 2, pp. 159171, Dec. 2014, doi: 10.2190/et.43.2.d.

[16] Andreas, C. R. Aldawira, H. W. Putra, N. Hanafiah, S. Surjarwo, and A. Wibisurya, "Door security system for home monitoring based on ESp32," Procedia Computer Science, vol. 157, pp. 673-682, 2019, doi: 10.1016/j.procs.2019.08.218

[17] A. Maier, A. Sharp, and Y. Vagapov, "Comparative analysis and practical implementation of the ESP32 microcontroller module for the internet of things," in 2017 Internet Technologies and Applications, ITA 2017 - Proceedings of the 7th International Conference, 2017, pp. 143-148, doi: 10.1109/ITECHA.2017.8101926.

[18] R. A, "Indoor Navigation System using BLE and ESP32," International Journal for Research in Applied Science and Engineering Technology, vol. 8, no. 11, pp. 151-159, Nov. 2020, doi: 10.22214/ijraset.2020.32089.

[19] S. Santos, "ESP32 Pinout Reference: Which GPIO pins should you use? | Random Nerd Tutorials," Random Nerd Tutorials, 2018. https://randomnerdtutorials.com/esp32-pinout-reference-gpios/ (accessed May 18, 2020).

[20] John Teel, "How to Set Up an ESP32 Wireless Microcontroller as a General Purpose Processor Using the Arduino IDE," 2021. https://www.electromaker.io/blog/article/esp32-wireless-microcontroller-as-a-general-purpose-processor-using-the-arduino-ide (accessed Jan. 29, 2021).

[21] A.-L. BOER, M.-C. LUCULESCU, L. CRISTEA, S.-C. ZAMFIRA, and I. BARBU, "Comparative Study Between Global Positioning Systems Used on Remotely Piloted Aircraft Systems," Scientific Research and Education in the Air Force, vol. 18, no. 1, pp. 127-132, Jun. 2016, doi: 10.19062/2247-3173.2016.18.1.16

[22] L. Lu, L. Ma, T. Wu, and X. Chen, "Performance analysis of positioning solution using low-cost single-frequency u-blox receiver based on baseline length constraint," Sensors (Switzerland), vol. 19, no. 19, p. 4352, Oct. 2019, doi: 10.3390/s19194352.

[23] T. Amanatidis and A. Chatzigeorgiou, "Studying the evolution of PHP web applications," Information and Software Technology, vol. 72, pp. 48-67, Apr. 2016, doi: 10.1016/j.infsof.2015.11.009.

[24] "What is phpMyAdmin: Tutorial, Features, Installation." https://www.javatpoint.com/phpmyadmin (accessed Feb. 10, 2021)

[25] A. K. Singh, D. A. Kumar, and V. Prakash, "Importance and Needs of IoT in Developing Smart Cities," SSRN Electronic Journal, 2019, doi: 10.2139/ssrn.3350286.

[26] A. Shapiro, "Why Do Unique IP Addresses Matter and What Is Their Importance? Business 2 Community." https://www.business2community.com/tech-gadgets/why-do-unique-ip-addresses-matter-and-what-is-their-importance-0474610 (accessed Apr. 24, 2013).

\section{BIOGRAPHIES OF AUTHORS}

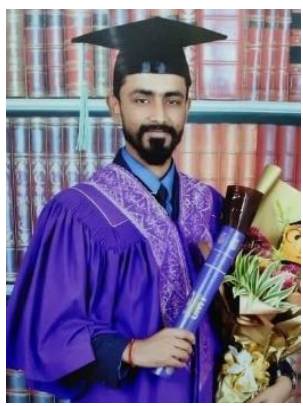

Arunkumar Subramaniam (iD) Sc sc P received the Bachelor of Applied Sciences (Electronic and Instrumentation Physics) from Universiti Malaysia Terengganu in 2019 and currently pursuing his Master Degree in Engineering and Engineering Studies (Electronics) in Universiti Malaysia Terengganu. He is also currently working as Project Engineer (Automation) at MAPA Gloves Sdn. Bhd. His main research interest is in instrumentation \& automation systems, control systems, communication protocols, energy management, driving cycle and implementation of IoT in big data management. He can be contacted at email: P4374@pps.umt.edu.my. ResearchGate: https://www.researchgate.net/profile/Arunkumar-Subramaniam.

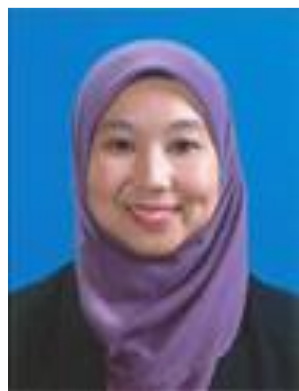

Nurru Anida Ibrahim (D) 81 sc $\mathrm{P}$ received the Bachelor of Engineering (Mechatronics) from International Islamic University Malaysia, Gombak in 2015 her and Master Degree in Physics (Renewable Energy) from Universiti Malaysia Terengganu in 2019. She currently pursuing her $\mathrm{Ph} . \mathrm{D}$ in Engineering (Energy and Electrical) Her main research interest is Plug in Hybrid Vehicle, driving cycle, energy rate, emissions and implementation of IoT in big data management. She can be contacted at email: P4620@pps.umt.edu.my. 

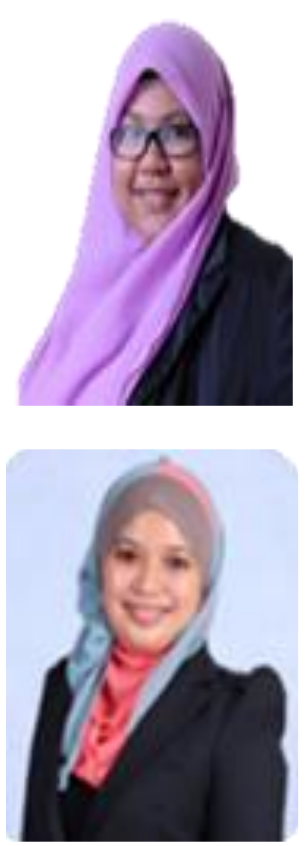

Siti Norbakyah Jabar (iD Io SC P received the Bachelor of Applied Science (Physics Electronic and Instrumentation) and $\mathrm{MSc}$ and $\mathrm{PhD}$ from Universiti Malaysia Terengganu, Terengganu, in 2009, 2014 and 2019 respectively. She is currently working as a lecturer at Universiti Malaysia Terengganu, Malaysia. Her main research interest is Electric Vehicle, Hybrid Electric Vehicle, Plug in Hybrid Vehicle, Modelling and simulation and renewable energy. She can be contacted at email: bakyahjabar@umt.edu.my. ResearchGate: https://www.researchgate.net/profile/Js-Norbakyah.

Salisa Abdul Rahman (D) 8 SC P received the B.E. and M.E. in Electrical \& Electronics Engineering from University of Technology Petronas, Perak, Malaysia in 2004 and 2006, respectively while Ph.D. in Optimal Energy Management Strategy for the University of Technology Sydney Plug-In Hybrid Electric Vehicles from University of Technology Sydney (UTS), Australia. She is currently working as a senior lecturer at University Malaysia Terengganu, Malaysia. Her research interests are in Hybrid Electric Vehicles, innovation powertrain, simulation and modeling, energy management strategy, driving cycles, fuel economy, emissions and optimization. She can be contacted at email: salisa@umt.edu.my. ResearchGate: https://www.researchgate.net/profile/Salisa-Abdul-Rahman. 